Supporting Information for

\title{
SpinCouple: Development of a web tool for analyzing metabolite mixtures via 2D $J$-resolved NMR database
}

\author{
Jun Kikuchi ${ }^{\dagger, \star, f^{,},}$, Yuuri Tsuboi ${ }^{\dagger}$, Keiko Komatsu ${ }^{\dagger}$, Masahiro Gomi ${ }^{\dagger}$, Eisuke Chikayama ${ }^{\dagger, \S}$, Yasuhiro Date ${ }^{\dagger,+}$
}

${ }^{\dagger}$ RIKEN Center for Sustainable Resource Science, 1-7-22 Suehiro-cho, Tsurumi-ku, Yokohama, Kanagawa 230-0045, Japan

${ }^{\ddagger}$ Graduate School of Medical Life Science, Yokohama City University, 1-7-29 Suehiro-cho, Tsurumi-ku, Yokohama, Kanagawa 230-0045, Japan

${ }^{\S}$ Department of Information Systems, Niigata University of International and Information Studies, 3-1-1 Mizukino, Nishi-ku, Niigata-shi, Niigata 950-2292, Japan

"Graduate School of Bioagricultural Sciences, Nagoya University, 1 Furo-cho, Chikusa-ku, Nagoya, Aichi 464-0810, Japan

Contents:

Figure S1. Representative screen shot for determination of signal assignments and metabolite structure (this case is propionate) by CMC-assist program (Bruker Biospin, Rheinstetten, Germany), assisted with information from $2 \mathrm{D}$-Jres $\left({ }^{1} \mathrm{H}\right.$ chemical shift and $J$-coupling pattern) and 1D-NMR (intensity).

Figure S2. Comparison between the peak number and distributions of standard compounds measured by SpinCouple (red) and obtained from BML (blue), plotted as 2D-Jres spectra (A). Histogram of all peaks $(>10,000)$ obtained from SpinCouple (red) and obtained from BML (blue) plotted as 0.5-ppm binned data, similar to a 1D-NMR spectrum.

Figure S3. Comparison of the ${ }^{1} \mathrm{H}$ chemical shift values (A), $J$-couplings (B), and intensities (C) obtained using SpinCouple and BML data for four types of metabolites (Glu; red, Gln; blue, Glc; green, Mal; orange).

Figure S4. Entity relationship diagram for the SpinCouple program. 
Figure S5. Histograms of counts of query hits using already-assigned biological extract data sets against more than 10,000 standard database peaks by inputting different tolerance values of $\delta^{1} \mathrm{H}(\mathrm{A})$ and $J(\mathrm{~B})$.

Figure S6. Representative calibration curves for absolute quantification of metabolite concentration from the 2D Jres data. Various colors and symbols highlighted in each figure are different peaks among isoleucine (A) and phenylalanine (B). All compounds used in this study are listed in Table S1.

Figure S7. (A) Bar graphs demonstrating the quantification of the known standard mixtures used in Fig. 3. Abbreviations and mixture compositions for mix 1 and mix 2 are listed in Table S2. Blue symbols indicate concentration $(\mathrm{mM})$, whereas filled-red symbols show the output values of the calibration-based quantification by the SpinCouple program. Open-red symbols indicate quantified concentrations obtained using commercial software (Chenomx). (B) Comparison of the spectral decomposition results obtained using Chenomx (left) and the skyline-plot of the 2D-Jres projection of mix 2 samples at approximately $1.32 \mathrm{ppm}$ (top) and $4.12 \mathrm{ppm}$ (bottom).

Table S1. List of 38 commonly observed standard compounds and their correlation coefficients from standard curves.

Table S2. Compositions of known 20 compound mixtures used for the demonstration of calibration curve-based quantification by SpinCouple (mM).

Table.S3 Full list of compounds deposited in SpinCouple 


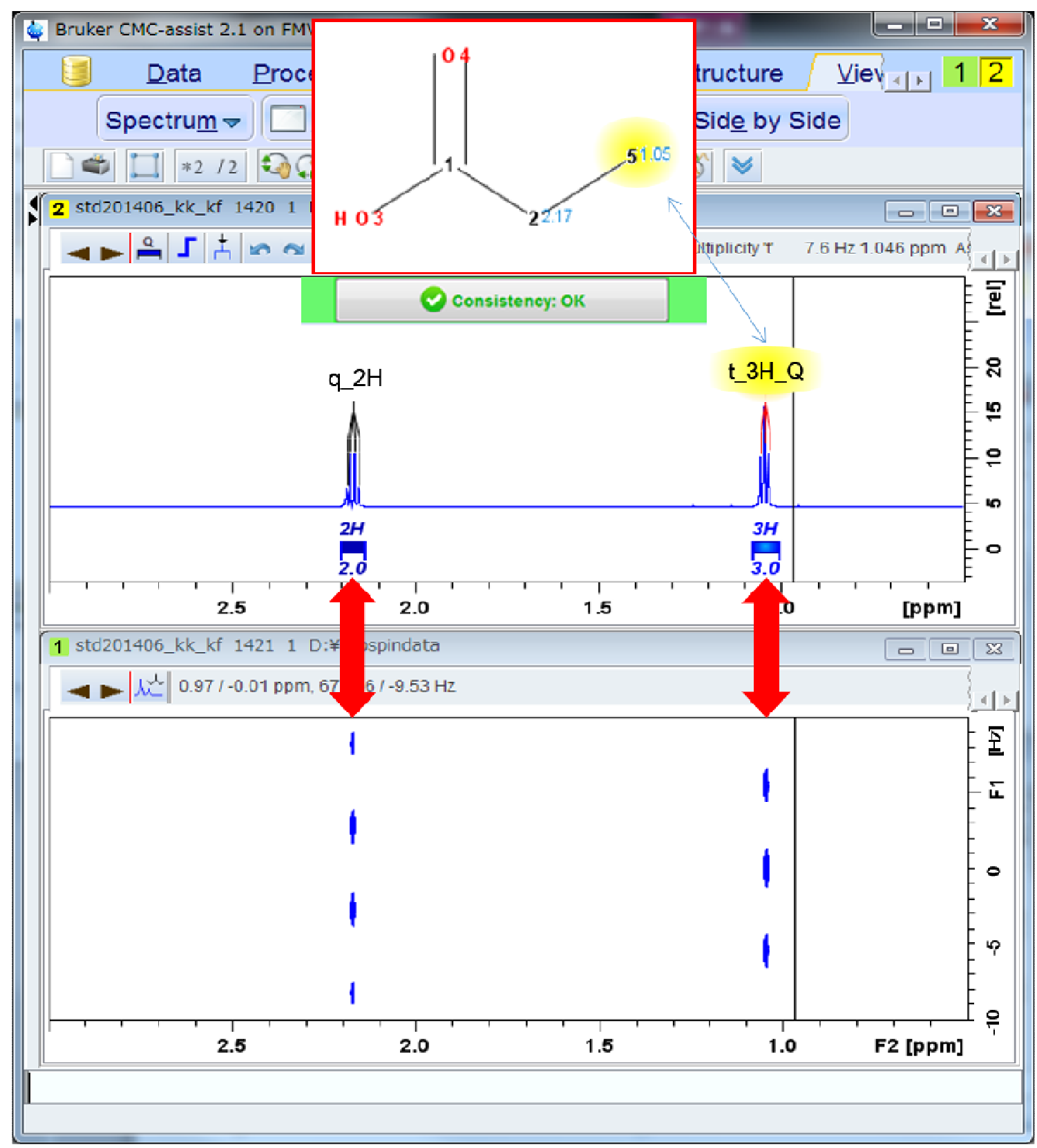

Figure S1. Representative screen shot for signal assignments and determination of metabolite structure (propionate) by CMC-assist program ((Bruker Biospin, Rheinstetten, Germany), assisted with information from 2D-Jres $\left({ }^{1} \mathrm{H}\right.$ chemical shift and $J$-coupling pattern) and 1D-NMR (intensity). 
(A)
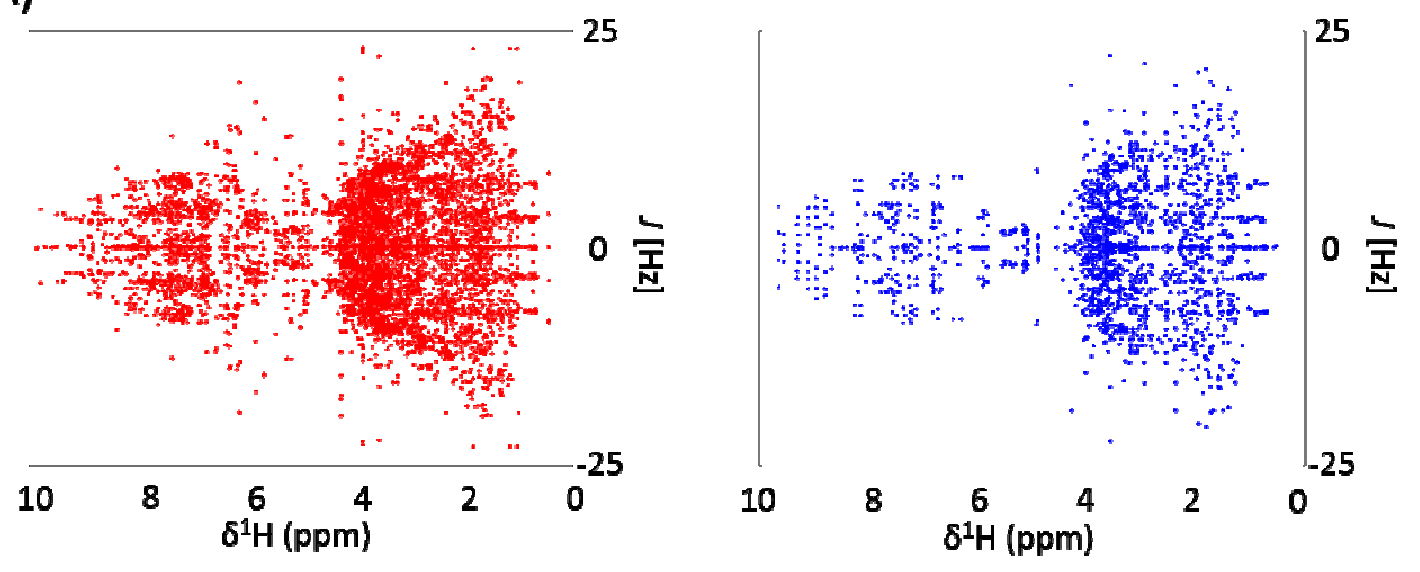

(B)

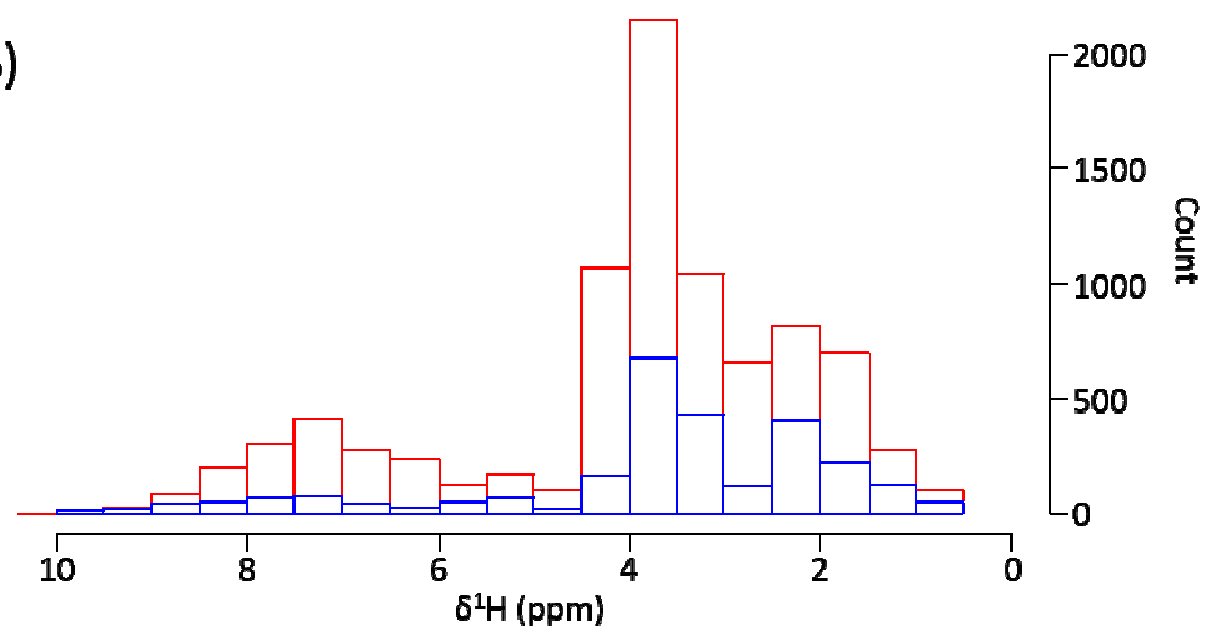

Figure S2. Comparison between the peak number and distributions of standard compounds measured by SpinCouple (red) and obtained from BML (blue) plotted as 2D-Jres spectra (A). Histogram of all peaks $(>10,000)$ SpinCouple (red) and obtained from BML (blue) plotted by $0.5 \mathrm{ppm}$ binned data like as 1D-NMR spectrum. 

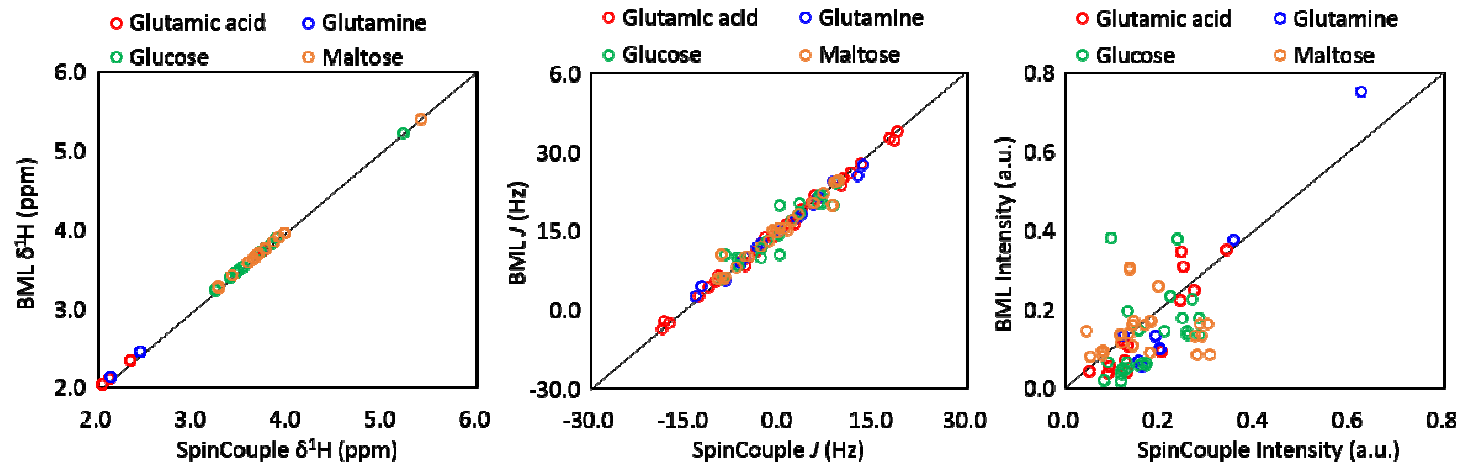

Figure S3. Comparison of ${ }^{1} \mathrm{H}$ chemical shift (A), $J$-coupling (B), and Intensities (C) between SpinCouple versus BML data among four types of metabolites (Glu: red, Gln: blue, Glc: green, Mal: orange). 


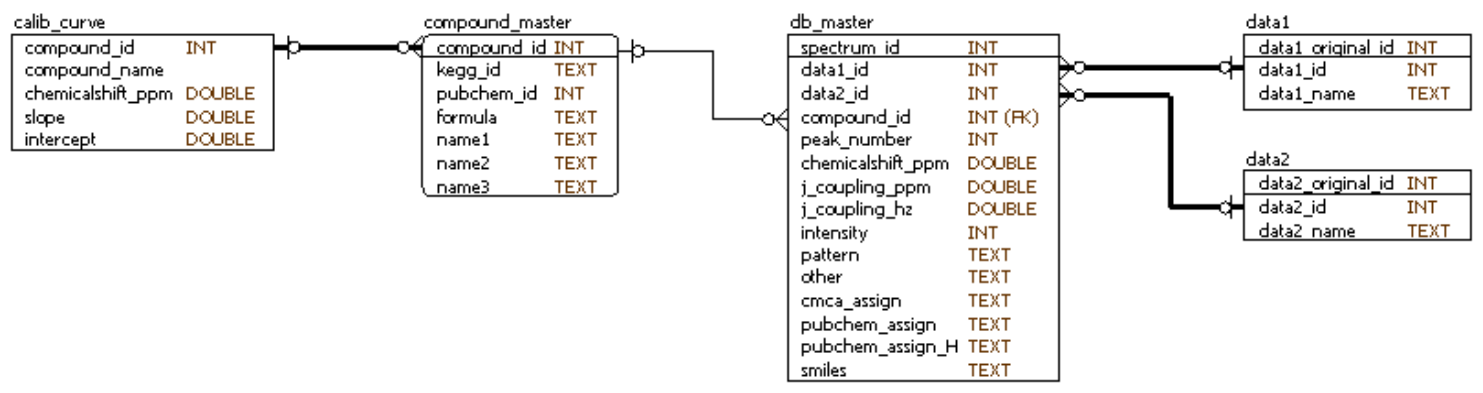

Figure S4. Entity relationship diagram for the SpinCouple program. 
(A)

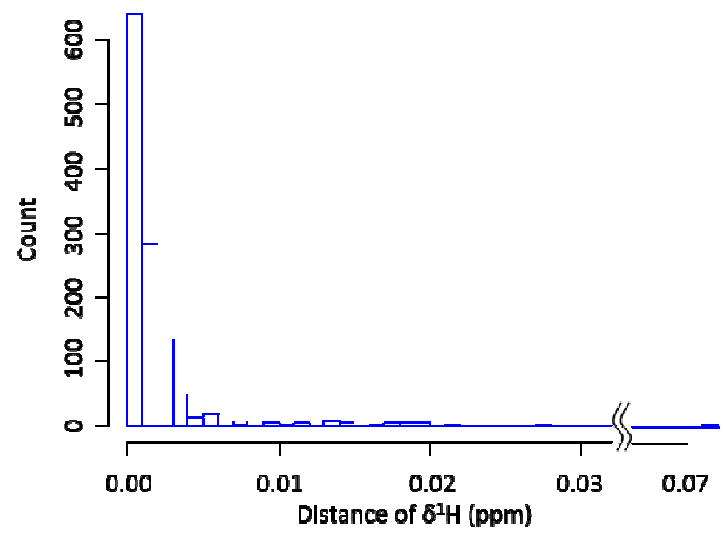

(B)

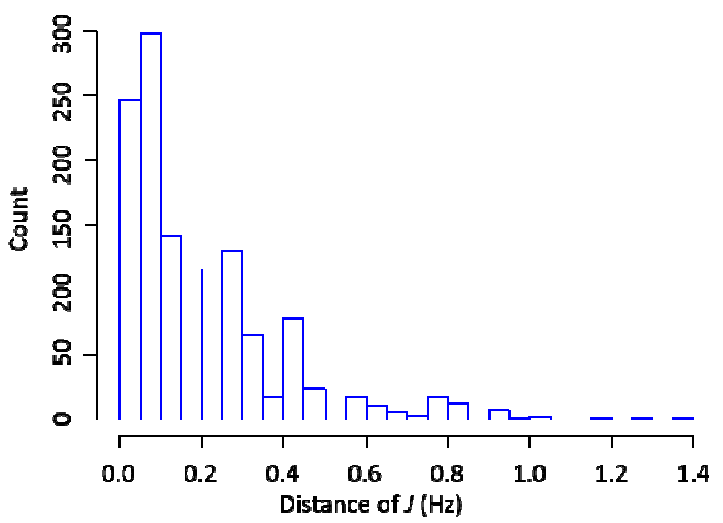

Figure S5. Histograms of counts of query hit using already assigned biological extract data sets against over 10,000 standard database peaks by inputting different tolerance values of $\delta^{1} \mathrm{H}(\mathrm{A})$ and $J(\mathrm{~B})$. 
(A)

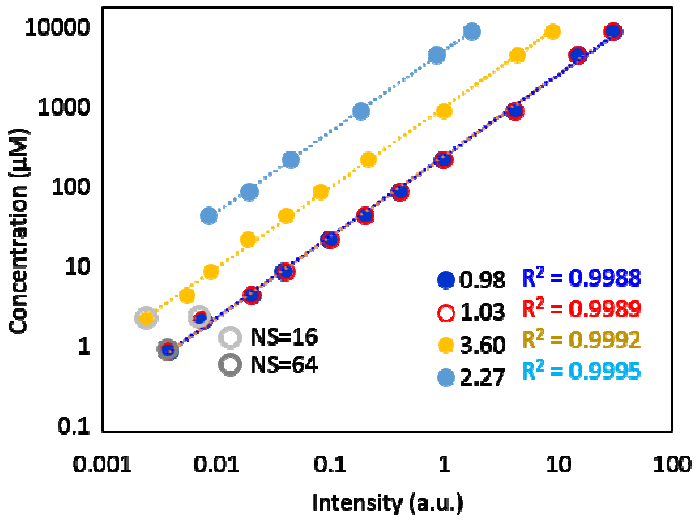

(B)

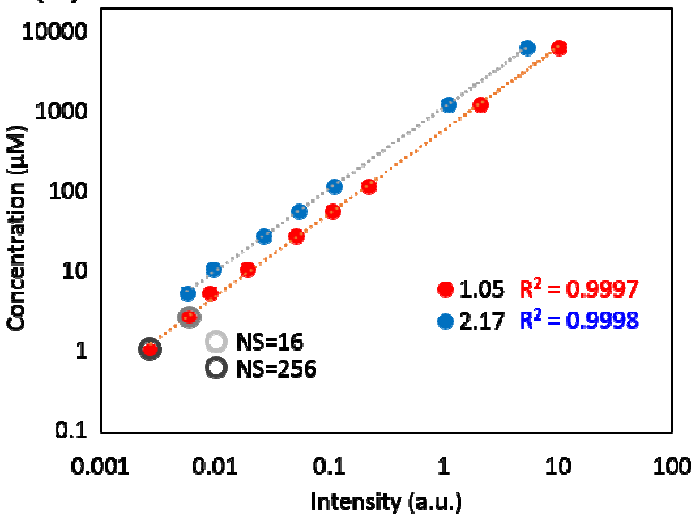

Figure S6. Representative calibration curves for absolute quantification of metabolite concentration from the 2D

Jres data. Various colors and symbols highlighted in each figure are different peaks among valine (A) and propionate (B). All compounds used in this study were listed in Table S1. 
(A)

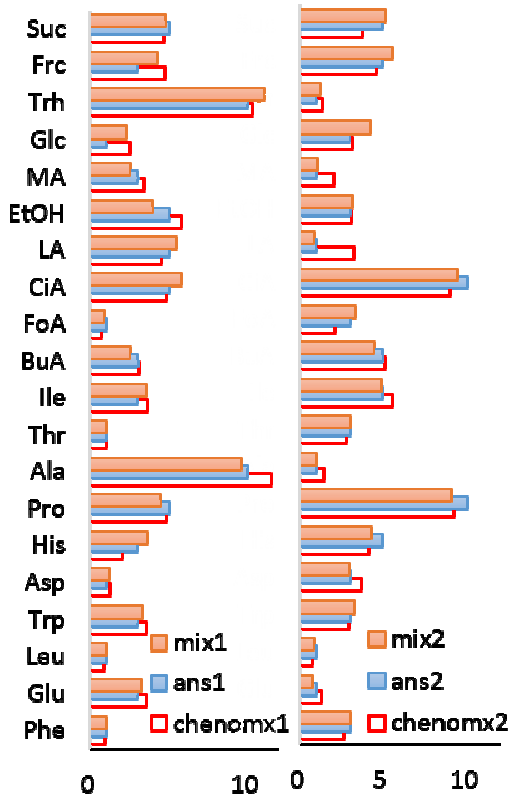

(B)

\section{Chenomx}

$1 \mathrm{mM}$ Lactate at $1.32 \mathrm{ppm}$

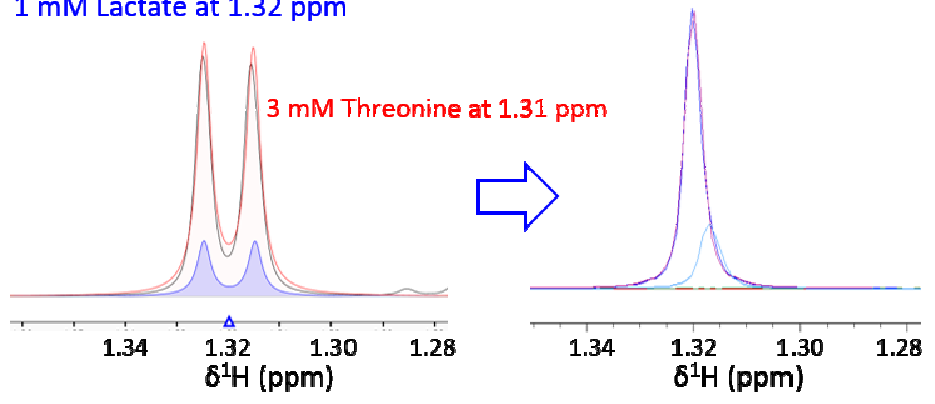

$1 \mathrm{mM}$ Lactate at $4.12 \mathrm{ppm}$

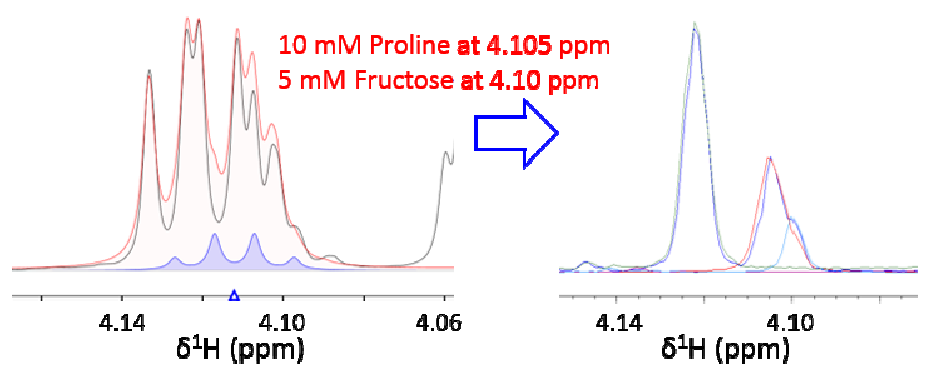

Figure S7. (A) Bar-graphs showing demonstration of quantification of known standard mixtures used in

Fig.3. Abbreviations and mixture compositions mix 1 and mix 2 are listed in Table S2. Blue indicated of the concentration $(\mathrm{mM})$, whereas filled red demonstrated output values calibration-based quantification by from SpinCouple. Open red showed quantified concentration using commercial software (Chenomx). (B) Comparison of spectral decomposition by Chenomx (left) and skyline-plot of 2D-Jres projection of mix2 samples around $1.32 \mathrm{ppm}$ (top) and $4.12 \mathrm{ppm}$ (bottom). 
Table S1. List of 38 commonly observed standard compounds and their correlation coefficients from standard curves.

\begin{tabular}{|c|c|c|c|c|c|c|c|}
\hline No. & Metabolites & $\begin{array}{l}\text { Chemical } \\
\text { shift (ppm) }\end{array}$ & $\mathrm{R}^{2}$ & No. & Metabolites & $\begin{array}{l}\text { Chemical } \\
\text { shift (ppm) }\end{array}$ & $\mathrm{R}^{2}$ \\
\hline \multirow[t]{4}{*}{1} & \multirow[t]{4}{*}{ Valine } & 0.98 & 0.9988 & 28 & Formate & 8.45 & 0.9932 \\
\hline & & 1.03 & 0.9989 & 29 & Methanol & 3.34 & 1.0000 \\
\hline & & 3.60 & 0.9992 & 30 & Ethanol & 1.17 & 0.9935 \\
\hline & & 2.27 & 0.9995 & & & 3.64 & 0.9923 \\
\hline \multirow[t]{3}{*}{2} & \multirow[t]{3}{*}{ Methionine } & 2.13 & 0.9999 & 31 & Raffinose & 3.67 & 0.9966 \\
\hline & & 2.63 & 0.9991 & & & 3.57 & 0.9989 \\
\hline & & 3.85 & 0.9976 & & & 3.74 & 0.9996 \\
\hline \multirow[t]{5}{*}{3} & \multirow[t]{5}{*}{ Isoleucine } & 1.00 & 0.9984 & & & 3.82 & 0.9989 \\
\hline & & 0.93 & 0.9985 & & & 3.77 & 0.9968 \\
\hline & & 3.66 & 0.9981 & & & 3.76 & 0.9987 \\
\hline & & 1.46 & 0.9980 & & & 4.22 & 0.9996 \\
\hline & & 1.25 & 0.9987 & & & 4.06 & 0.9986 \\
\hline \multirow[t]{3}{*}{4} & \multirow[t]{3}{*}{ Threonine } & 1.32 & 0.9992 & & & 5.42 & 0.9974 \\
\hline & & 3.58 & 0.9985 & & & 4.99 & 0.9963 \\
\hline & & 4.25 & 0.9968 & & & 3.89 & 0.9991 \\
\hline \multirow[t]{6}{*}{5} & \multirow[t]{6}{*}{ Leucine } & 0.96 & 0.9965 & & & 3.83 & 0.9984 \\
\hline & & 0.95 & 0.9951 & & & 4.00 & 0.9913 \\
\hline & & 1.74 & 0.9881 & & & 3.95 & 0.9798 \\
\hline & & 3.73 & 0.9959 & 32 & Trehalose & 3.64 & 0.9967 \\
\hline & & 1.70 & 0.9844 & & & 5.19 & 0.9915 \\
\hline & & 1.68 & 0.9898 & & & 3.85 & 0.9910 \\
\hline \multirow[t]{8}{*}{6} & \multirow[t]{8}{*}{ Tryptophan } & 7.32 & 0.9943 & & & 3.76 & 0.9934 \\
\hline & & 4.05 & 0.9960 & & & 3.44 & 0.9965 \\
\hline & & 3.30 & 0.9947 & 33 & Cellobiose & 3.50 & 0.9993 \\
\hline & & 3.48 & 0.9944 & & & 3.31 & 0.9988 \\
\hline & & 7.19 & 0.9961 & & & 3.42 & 0.9965 \\
\hline & & 7.73 & 0.9961 & & & 3.50 & 0.9993 \\
\hline & & 7.28 & 0.9974 & & & 3.57 & 0.9986 \\
\hline & & 7.54 & 0.9971 & & & 3.28 & 0.9967 \\
\hline \multirow[t]{2}{*}{7} & \multirow[t]{2}{*}{ Glutamine } & 3.76 & 0.9999 & & & 3.48 & 0.9935 \\
\hline & & 2.43 & 0.9997 & & & 3.73 & 0.9989 \\
\hline
\end{tabular}




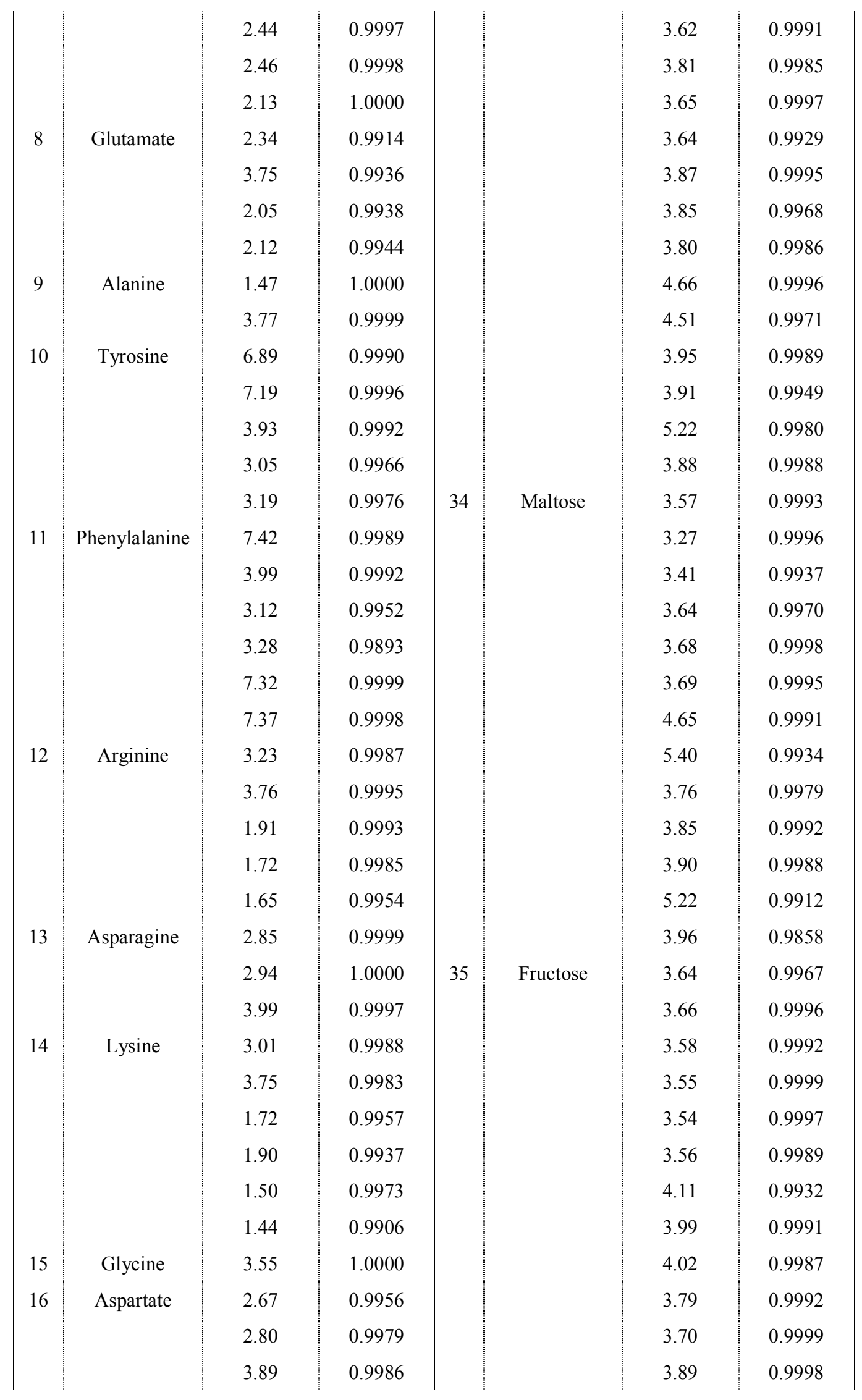




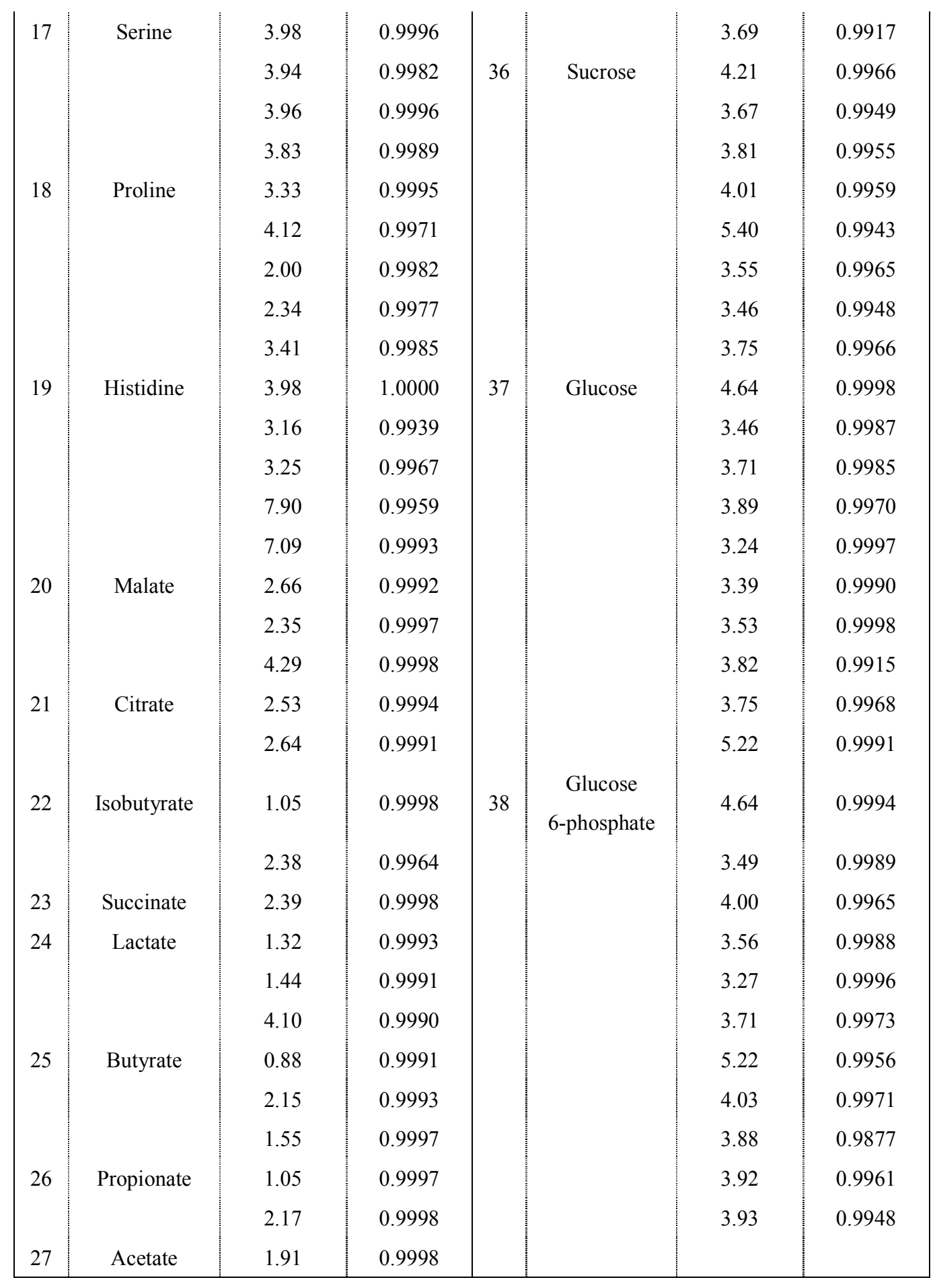


Table S2. Compositions of known 20-compound mixtures used for the demonstration of calibration curve-based quantification by SpinCouple (mM). Black, blue, and green text indicate amino acids, organic acids and ethanol, and saccharides, respectively; the listed abbreviations are also used in Fig. S3.

\begin{tabular}{|l|cccc|}
\hline Metabolites & mix1 & mix2 & mix3 & mix4 \\
\hline Phenylalanine (Phe) & 1 & 3 & 5 & 3 \\
Glutamate (Glu) & 3 & 1 & 3 & 1 \\
Leucine (Leu) & 1 & 1 & 3 & 3 \\
Tryptophan (Trp) & 3 & 3 & 1 & 1 \\
Aspartate (Asp) & 1 & 3 & 1 & 3 \\
Histidine (His) & 3 & 5 & 5 & 1 \\
Proline (Pro) & 5 & 10 & 1 & 3 \\
Alanine (Ala) & 10 & 1 & 3 & 5 \\
Threonine (Thr) & 1 & 3 & 5 & 3 \\
Isoleucine (Ile) & 3 & 5 & 10 & 1 \\
Butyrate (BuA) & 3 & 5 & 3 & 1 \\
Formate (FoA) & 1 & 3 & 5 & 10 \\
Citrate (CiA) & 5 & 10 & 1 & 3 \\
Lactate (LA) & 5 & 1 & 3 & 5 \\
Ethanol (EtOH) & 5 & 3 & 1 & 3 \\
Malate (MA) & 3 & 1 & 3 & 5 \\
Glucose (Glc) & 1 & 3 & 5 & 10 \\
Trehalose (Trh) & 10 & 1 & 3 & 5 \\
Fructose (Frc) & 3 & 5 & 3 & 1 \\
Sucrose (Suc) & 5 & 5 & 1 & 3 \\
\hline
\end{tabular}


Table S3 Full list of compounds deposited in SpinCouple

Data1_list

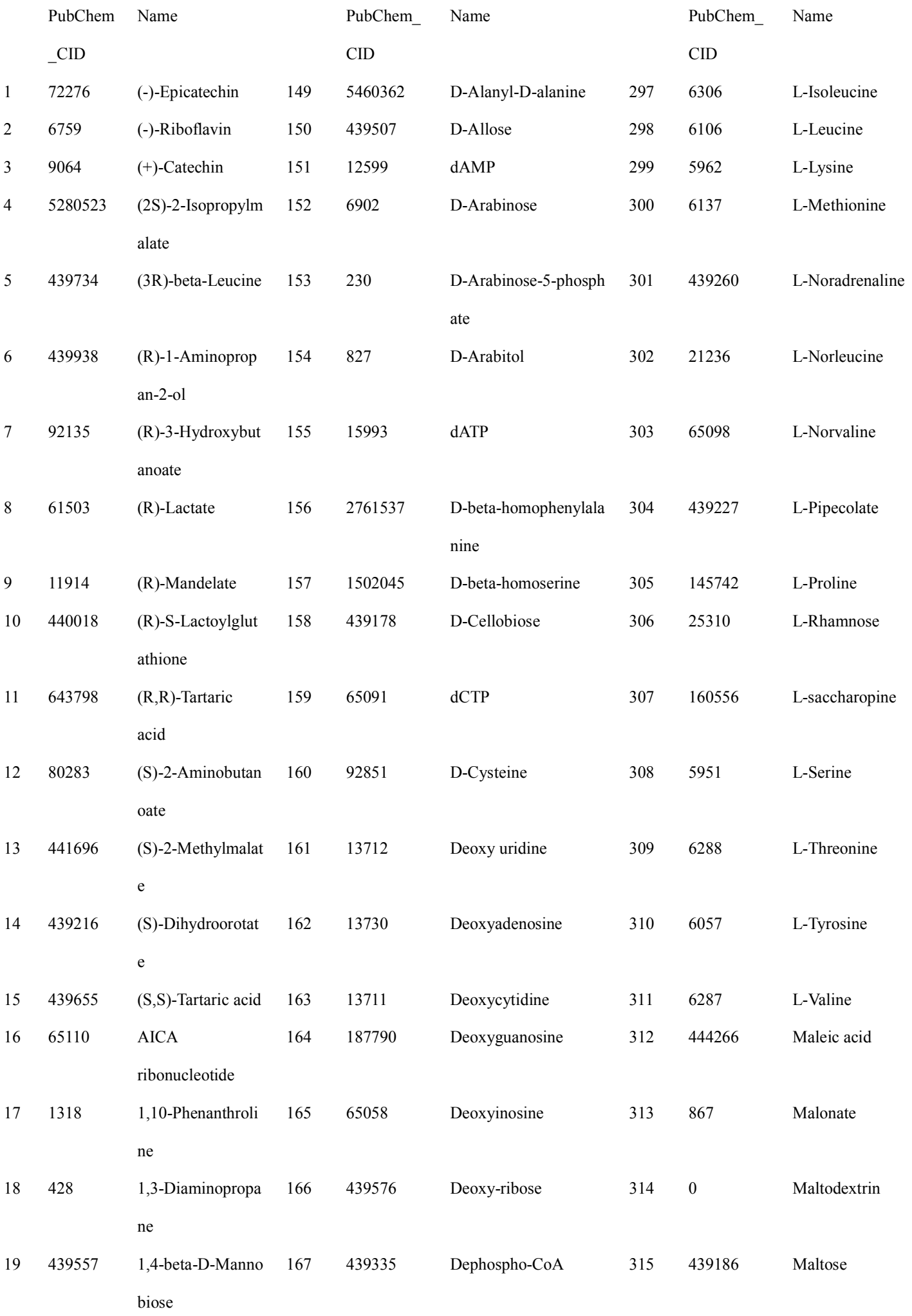




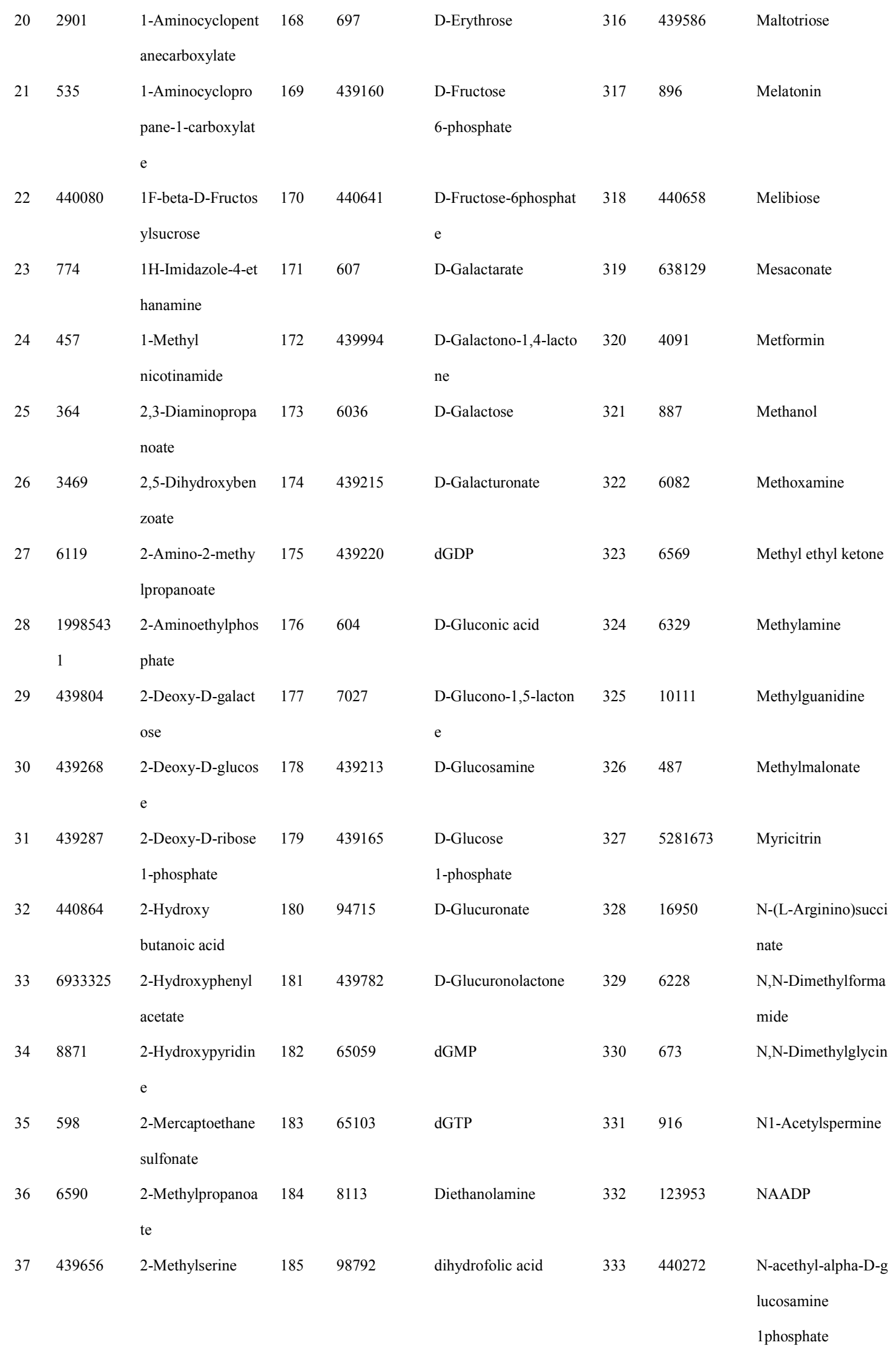




\begin{tabular}{|c|c|c|c|c|c|c|c|c|}
\hline 38 & 398 & 2-Nitropropane & 186 & 91531 & dIMP & 334 & 439174 & $\begin{array}{l}\text { N-Acetyl-D-glucosa } \\
\text { mine }\end{array}$ \\
\hline 39 & 58 & 2-Oxobutanoate & 187 & 6 & Dinitrochlorobenzene & 335 & 439219 & $\begin{array}{l}\text { N-Acetyl-D-glucosa } \\
\text { mine 6-phosphate }\end{array}$ \\
\hline 40 & 74563 & $\begin{array}{l}\text { 2-Oxopentanoic } \\
\text { acid }\end{array}$ & 188 & 94206 & D-Isoleucine & 336 & 439281 & $\begin{array}{l}\text { N-Acetyl-D-mannos } \\
\text { amine }\end{array}$ \\
\hline 41 & 127 & $\begin{array}{l}\text { 3-(4-Hydroxyphen } \\
\text { yl)acetate }\end{array}$ & 189 & 146302 & dITP & 337 & 10972 & N-Acetylglycine \\
\hline 42 & 979 & $\begin{array}{l}\text { 3-(4-Hydroxyphen } \\
\text { yl)pyruvate }\end{array}$ & 190 & 441441 & $\begin{array}{l}\text { DL-2-Aminobutyric } \\
\text { acid }\end{array}$ & 338 & 65065 & $\begin{array}{l}\text { N-Acetyl-L-aspartat } \\
\text { e }\end{array}$ \\
\hline 43 & 72 & $\begin{array}{l}\text { 3,4-Dihydroxyben } \\
\text { zoate }\end{array}$ & 191 & 64956 & $\begin{array}{l}\text { DL-3-Aminoisobutyric } \\
\text { acid }\end{array}$ & 339 & 70914 & $\begin{array}{l}\text { N-Acetyl-L-glutama } \\
\text { te }\end{array}$ \\
\hline 44 & 6047 & $\begin{array}{l}\text { 3,4-Dihydroxy-L- } \\
\text { phenylalanine }\end{array}$ & 192 & 2724399 & $\begin{array}{l}\text { DL-5-Hydroxylysine } \\
\text { hydrochloride }\end{array}$ & 340 & 439197 & $\begin{array}{l}\text { N-Acetylneuraminat } \\
\text { e }\end{array}$ \\
\hline 45 & 85782 & $\begin{array}{l}\text { 3,4-Dihydroxyma } \\
\text { ndelate }\end{array}$ & 193 & 439283 & $\begin{array}{l}\text { DL-alpha,epsilon-Dia } \\
\text { minopimelic acid }\end{array}$ & 341 & 5893 & NAD \\
\hline 46 & 547 & $\begin{array}{l}\text { 3,4-Dihydroxyphe } \\
\text { nylacetate }\end{array}$ & 194 & 185698 & D-Mannose & 342 & 92794 & $\begin{array}{l}\text { Naringenin } \\
\text { 7-O-beta-D-glucosid } \\
\text { e }\end{array}$ \\
\hline 47 & 6076 & 3',5'-Cyclic AMP & 195 & 439198 & $\begin{array}{l}\text { D-Mannose } \\
\text { 6-phophate }\end{array}$ & 343 & 93072 & $\begin{array}{l}\mathrm{N} \text {-Carbamoyl-L-asp } \\
\text { artate }\end{array}$ \\
\hline 48 & 440384 & $\begin{array}{l}\text { 3alpha,7alpha-Dih } \\
\text { ydroxy-5beta-chol } \\
\text { estanate }\end{array}$ & 196 & 439297 & D-panose & 344 & 439750 & $\begin{array}{l}\text { N-Formyl-L-methio } \\
\text { nine }\end{array}$ \\
\hline 49 & 1639 & $\begin{array}{l}\text { 3-Amino-1,2,4-tri } \\
\text { azole }\end{array}$ & 197 & 164619 & D-Pinitol & 345 & 936 & Nicotinamide \\
\hline 50 & 41211 & 3'-AMP & 198 & 439242 & D-Raffinose & 346 & 14181 & $\begin{array}{l}\text { Nicotinamide } \\
\text { D-ribonucleotide }\end{array}$ \\
\hline 51 & 66535 & 3'-CMP & 199 & 16219932 & D-Ribose-5-phosphate & 347 & 16219767 & $\begin{array}{l}\text { Nicotinamide } \\
\text { hypoxanthine } \\
\text { dinucleotide }\end{array}$ \\
\hline 52 & 67701 & $\begin{array}{l}\text { 3-Guanidinopropa } \\
\text { noate }\end{array}$ & 200 & 5780 & D-Sorbitol & 348 & 938 & Nicotinate \\
\hline 53 & 5281167 & 3-Hexenol & 201 & 439304 & D-Sorbose & 349 & 121992 & $\begin{array}{l}\text { Nicotinate } \\
\text { D-ribonucleotide }\end{array}$ \\
\hline 54 & 1662 & $\begin{array}{l}\text { 3-Hydroxy-3-met } \\
\text { hylglutarate }\end{array}$ & 202 & 439312 & D-Tagatose & 350 & 3614 & N-Methylhistamine \\
\hline 55 & 802 & 3-Indoleacetic & 203 & 164628 & dTDP & 351 & 5288725 & N-Methyl-L-alanine \\
\hline
\end{tabular}




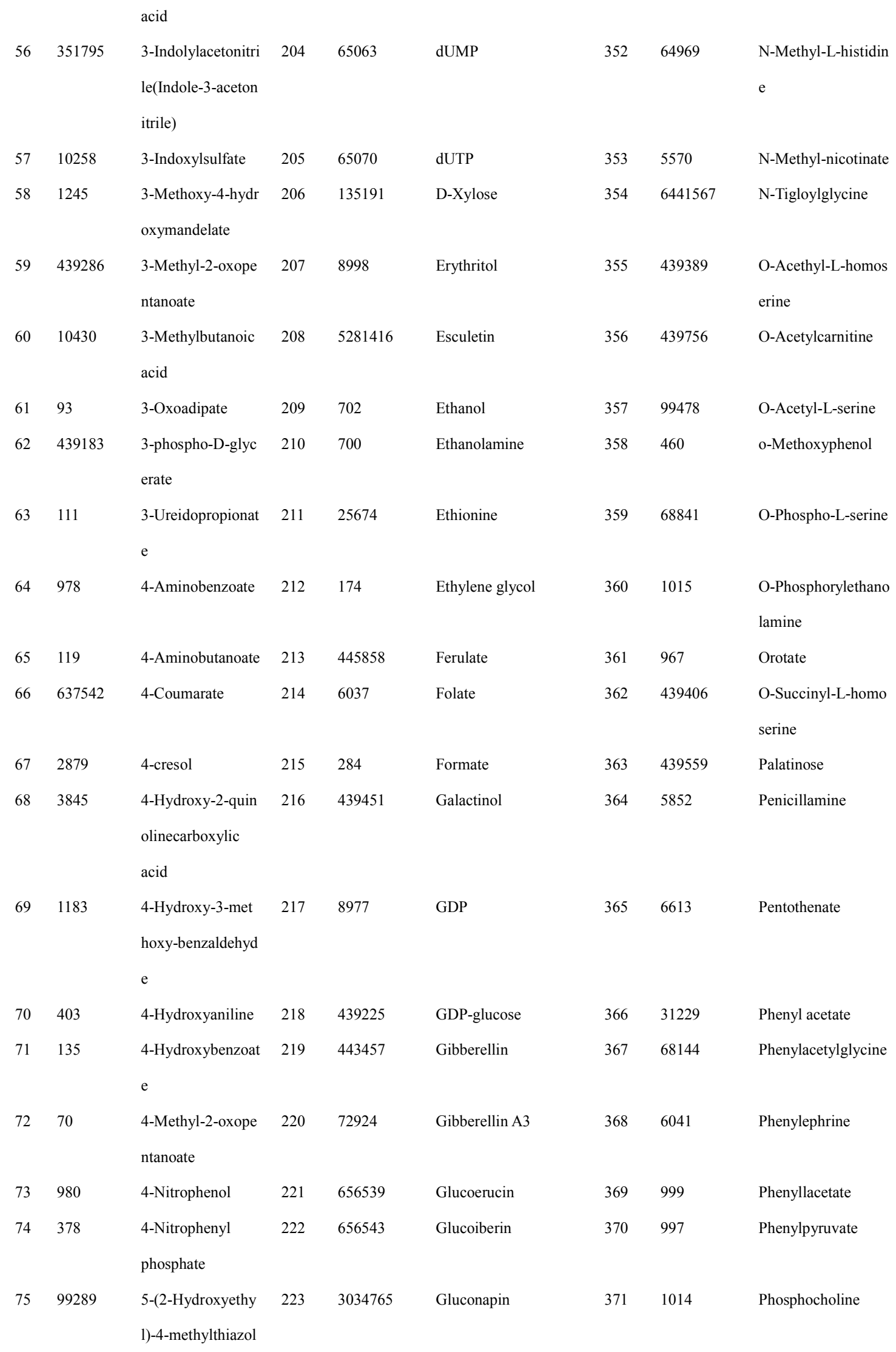




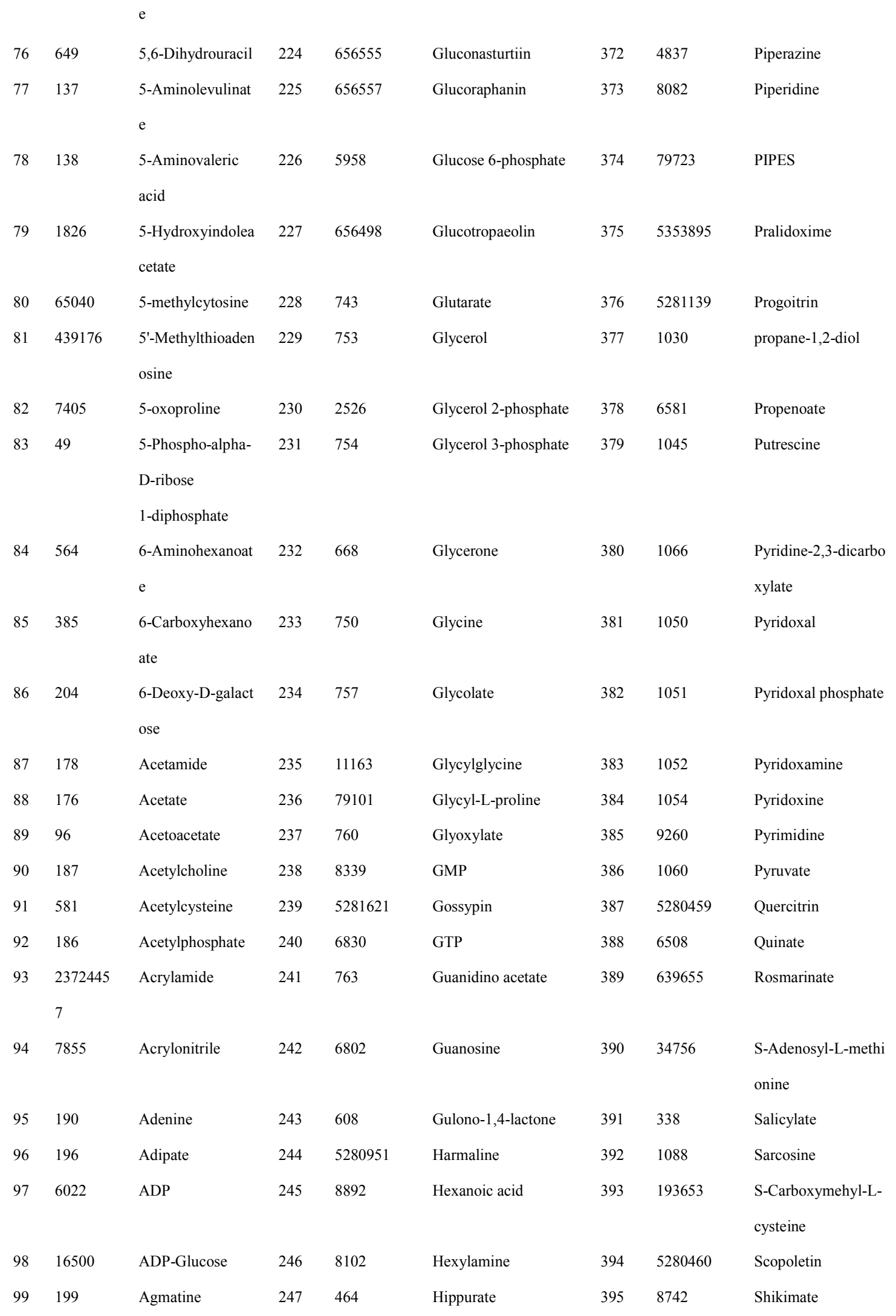




\begin{tabular}{|c|c|c|c|c|c|c|c|c|}
\hline 10 & 4901 & Alginate & 248 & 773 & Histidine & 396 & 656568 & Sinalbin \\
\hline \multicolumn{9}{|l|}{0} \\
\hline 10 & 203 & Allantoate & 249 & 89235 & Homocarnosine & 397 & 637775 & Sinapate \\
\hline \multicolumn{9}{|l|}{1} \\
\hline 10 & 123912 & alpha-D-Galactose & 250 & 780 & Homogentisate & 398 & 5280802 & Sinapoyl aldehyde \\
\hline 2 & & 1-phosphate & & & & & & \\
\hline 10 & 90638 & alpha-Methyl-DL- & 251 & 1738 & Homovanillate & 399 & 2723733 & Sinigrine \\
\hline 3 & & histidine & & & & & & \\
\hline 10 & 2130 & Amantadine & 252 & 107812 & Hypotaurine & 400 & 6549 & S-Methyl-L-cysteine \\
\hline \multicolumn{9}{|l|}{4} \\
\hline 10 & 286 & Aminooxyacetic & 253 & 790 & Hypoxanthine & 401 & 439285 & sn-glycero-3-Phosph \\
\hline 5 & & acid & & & & & & ocholine \\
\hline 10 & 6083 & AMP & 254 & 8582 & IMP & 402 & 1102 & Spermidine \\
\hline \multicolumn{9}{|l|}{6} \\
\hline 10 & 6115 & Aniline & 255 & 798 & Indole & 403 & 439531 & Stachyose \\
\hline \multicolumn{9}{|l|}{7} \\
\hline 10 & 227 & Anthranilate & 256 & 800 & Indole-3-acetaldehyde & 404 & 10457 & Suberic acid \\
\hline \multicolumn{9}{|l|}{8} \\
\hline 10 & 5957 & АTP & 257 & 397 & Indole-3-acetamide & 405 & 1110 & Succinate \\
\hline \multicolumn{9}{|c|}{9} \\
\hline 11 & 240 & Benzaldehyde & 258 & 10256 & Indole-3-carboxaldehy & 406 & 5988 & Sucrose \\
\hline 0 & & & & & de & & & \\
\hline 11 & 2332 & Benzamidine & 259 & 69867 & Indole-3-carboxylic & 407 & 5316860 & Syringin \\
\hline 1 & & & & & acid & & & \\
\hline 11 & 243 & Benzoate & 260 & 3712 & Indole-3-methanol & 408 & 1123 & Taurine \\
\hline \multicolumn{9}{|l|}{2} \\
\hline 11 & 239 & beta-Alanine & 261 & 6021 & Inosine & 409 & 1130 & Thiamin \\
\hline \multicolumn{9}{|c|}{3} \\
\hline 11 & 112072 & beta-Alanyl-N(pi) & 262 & 6831 & Inosine-5'-diphosphate & 410 & 1131 & Thiamin \\
\hline 4 & & -methyl-L-histidin & & & & & & monophosphate \\
\hline & & 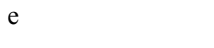 & & & & & & \\
\hline 11 & 1647 & beta-Aminopropio & 263 & 3765 & Isoguvacine & 411 & 1132 & Thiamine \\
\hline 5 & & nitrile & & & & & & diphosphate \\
\hline 11 & 64689 & beta-D-Glucose & 264 & 439193 & Isomaltose & 412 & 10484 & Thioacetate \\
\hline 6 & & & & & & & & \\
\hline 11 & 441422 & beta-Gentiobiose & 265 & 3767 & Isoniazid & 413 & 1133 & Thioglycolate \\
\hline 7 & & & & & & & & \\
\hline 11 & 248 & Betaine & 266 & 5922 & Isonicotinic acid & 414 & 439535 & Threonate \\
\hline
\end{tabular}


$$
0
$$

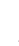

$$
12
$$$$
2
$$

12

12
4

5

6

12
7

8

9

0

13

1

\begin{tabular}{|c|c|c|c|c|c|c|c|c|}
\hline 13 & 311 & Citric Acid & 280 & 52940098 & L-beta-homoleucine & 428 & 1150 & Tryptamine \\
\hline \multicolumn{9}{|l|}{2} \\
\hline 13 & 6131 & CMP & 281 & 53397915 & L-beta-homolysine & 429 & 6305 & Tryptophan \\
\hline \multicolumn{9}{|l|}{3} \\
\hline 13 & 1549095 & Coniferyl alcohol & 282 & 13048315 & L-beta-homophenylala & 430 & 5610 & Tyramine \\
\hline 4 & & & & & nine & & & \\
\hline 13 & 5280536 & Coniferyl & 283 & 2761540 & L-beta-homoproline & 431 & 6031 & UDP \\
\hline 5 & & aldehyde & & & & & & \\
\hline 13 & 586 & Creatine & 284 & 1502076 & L-beta-homoserine & 432 & 445675 & UDP-acetylglucosa \\
\hline 6 & & & & & & & & mine \\
\hline 13 & 588 & Creatinine & 285 & 53398047 & L-beta-homothionine & 433 & 8629 & UDP-Glucose \\
\hline
\end{tabular}




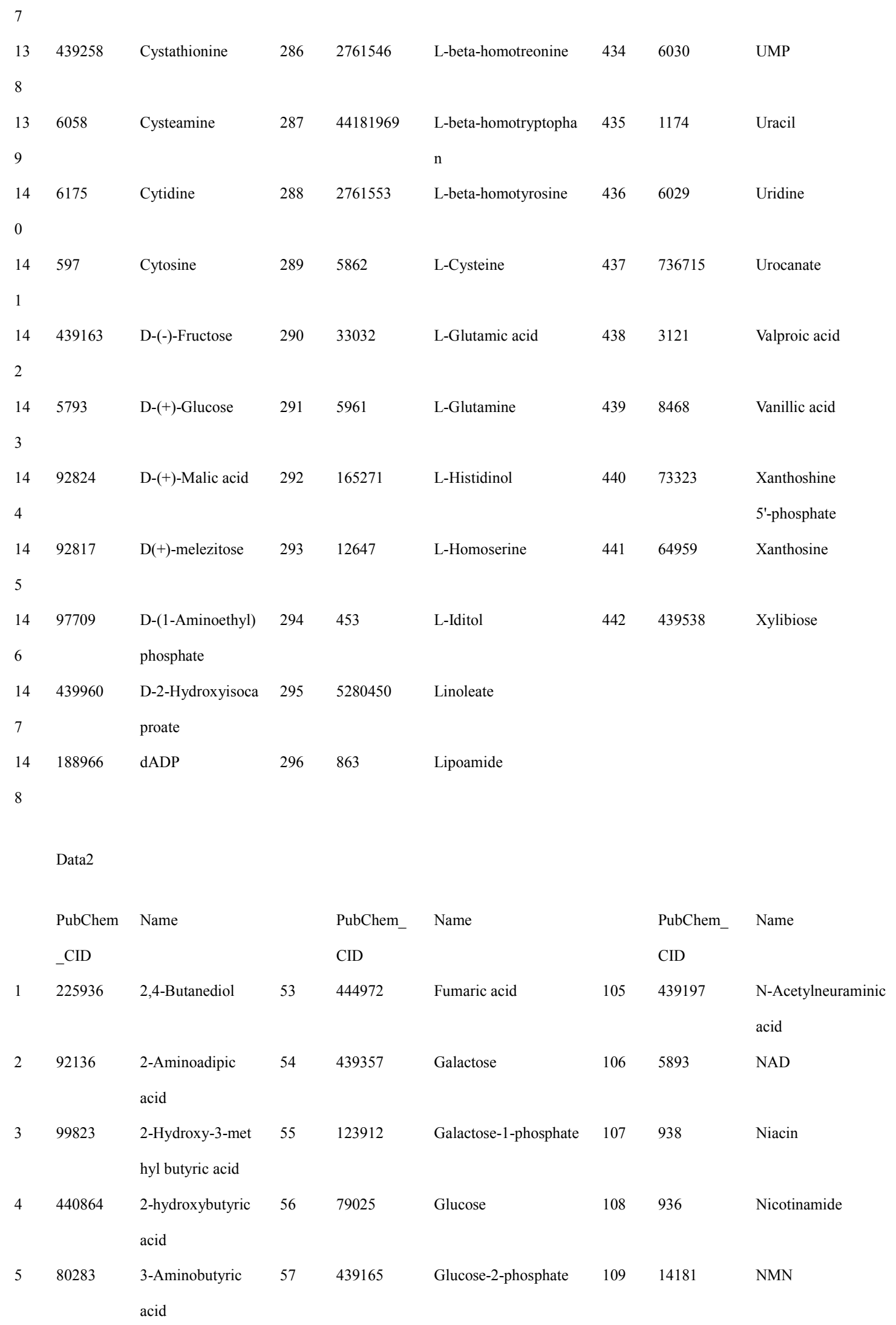




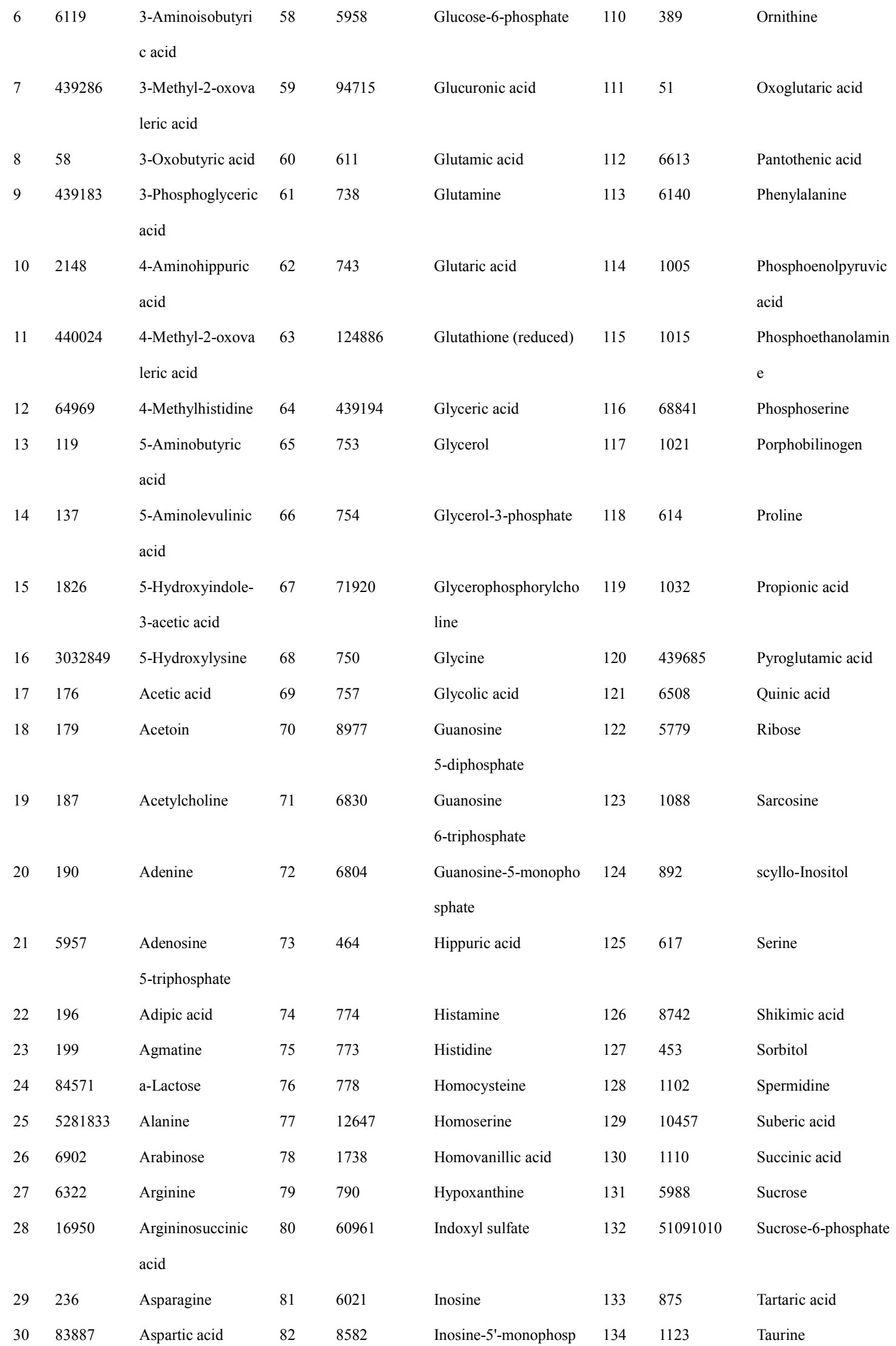




\begin{tabular}{|c|c|c|c|c|c|c|c|c|}
\hline & & & & & hate & & & \\
\hline 31 & 248 & Betaine & 83 & 7866 & Isethionic acid & 135 & 440567 & Taurocholic acid \\
\hline 32 & 264 & Butyric acid & 84 & 6590 & Isobutyric acid & 136 & 1130 & Thiamine \\
\hline 33 & 2969 & Capric acid & 85 & 1198 & Isocitric acid & 137 & 6288 & Threonine \\
\hline 34 & 10918 & Carnitine & 86 & 791 & Isoleucine & 138 & 5810 & trans-5-Hydroxyprol \\
\hline & & & & & & & & ine \\
\hline 35 & 439224 & Carnosine & 87 & 10430 & Isovaleric acid & 139 & 444212 & trans-Aconitic acid \\
\hline 36 & 221493 & Cholic Acid & 88 & 846 & Kynurenine & 140 & 444539 & trans-Cinnamic acid \\
\hline 37 & 305 & Choline & 89 & 61503 & Lactic acid & 141 & 7427 & Trehalose \\
\hline 38 & 643757 & cis-Aconitic acid & 90 & 6106 & Leucine & 142 & 122336 & Trehalose-6-phosph \\
\hline & & & & & & & & ate \\
\hline 39 & 311 & Citric acid & 91 & 864 & Lipoic acid & 143 & 5570 & Trigonelline \\
\hline 40 & 9750 & Citrulline & 92 & 866 & Lysine & 144 & 1146 & Trimethylamine \\
\hline 41 & 586 & Creatine & 93 & 444266 & Maleic acid & 145 & 1145 & Trimethylamine-N-o \\
\hline & & & & & & & & xide \\
\hline 42 & 588 & Creatinine & 94 & 525 & Malic acid & 146 & 6305 & Tryptophan \\
\hline 43 & 6058 & Cysteamine & 95 & 439186 & Maltose & 147 & 1153 & Tyrosine \\
\hline 44 & 594 & Cysteine & 96 & 18950 & Mannose & 148 & 8629 & Uridine \\
\hline & & & & & & & & 5-diphosphoglucose \\
\hline 45 & 6175 & Cytidine & 97 & 887 & Methanol & 149 & 6030 & Uridine \\
\hline & & & & & & & & 5-monophosphate \\
\hline 46 & 674 & Dimethylamine & 98 & 6137 & Methionine & 150 & 6133 & Uridine \\
\hline & & & & & & & & 5-triphosphate \\
\hline 47 & 700 & Ethanolamine & 99 & 6329 & Methylamine & 151 & 17473 & Uridine-5-diphospho \\
\hline & & & & & & & & glucuronic acid \\
\hline 48 & 643975 & FAD & 100 & 673 & N,N-Dimethylglycine & 152 & 736715 & Urocanic acid \\
\hline 49 & 6037 & Folic acid & 101 & 76406 & N-Acetylalanine & 153 & 7991 & Valeric acid \\
\hline 50 & 284 & Formic acid & 102 & 440272 & $\mathrm{~N}$-acetylglucosamine- & 154 & 827 & Xylitol \\
\hline & & & & & 1-phosphate & & & \\
\hline 51 & 439163 & Fructose & 103 & 439174 & N-Acetylglutamine & 155 & 135191 & Xylose \\
\hline 52 & 17106 & Fucose & 104 & 92832 & N-Acetyllysine & & & \\
\hline
\end{tabular}

